\section{On the Control of Chaotic Systems in Lur'e Form by Using Dither}

Ömer Morgül

\begin{abstract}
In this paper we propose the application of dither for controlling chaotic systems in the Lur'e form. Dither is a high-frequency periodic signal and has the effect of modifying the nonlinearity for some nonlinear systems. We use piecewise constant dither signals and propose three different methods for the selection of dither parameters. We also present some experimental results.
\end{abstract}

Index Terms - Chaos, control of chaos, dither, Lur'e systems.

\section{INTRODUCTION}

Recently, there has been an extensive interest on the study of analysis and control of chaotic behavior in nonlinear systems. Various control problems can be defined for such systems, such as targeting the trajectories to a desired point, stabilizing unstable periodic orbits, [1], etc. For an extensive list of references on this subject, see [2]. In this paper we propose a technique which may enable us to switch between the chaotic and regular (e.g., periodic) trajectories of some systems.

We consider the systems in the Lur'e form and propose the application of dither to control the behavior of such systems (see Fig. 1). Dither is a high-frequency periodic signal and has the effect of modifying the nonlinear characteristics by sweeping quickly across the domain of the nonlinear element. Such signals are typically used in nonlinear systems for the purpose of stabilization or elimination of limit cycles, or quenching undesirable jump phenomena (see [5] and [7],). If the power spectrum of dither lies above the cut-off frequency of the linear block (i.e., $L(s)$ in Fig. 1), it is filtered out before reaching the system output. However, it can be shown that prior to being filtered out, it modifies the nonlinearity (i.e., $n(\cdot)$ in Fig. 1) (see [5]-[7]). By using this property, it may be possible to control such a system if its behavior (e.g., chaos, limit cycle, etc.) is known in terms of the parameters of the nonlinear block, provided that those parameters could be modified by using dither. We may also use a conjecture proposed by Genesio and Tesi for the prediction of chaos in Lur'e-type systems (see [3] and [4]). By using these ideas we propose three methods to select the appropriate dither parameters to control the behavior of a given Lur'e system.

This paper is organized as follows. In Section II we outline the effect of dither and propose three methods to select appropriate dither parameters. In Section III we present some experimental results. Finally, we give some concluding remarks.

\section{Dither Control of Chaotic Systems}

Consider the system given in Fig. 1. Here, $L(s)$ represents the transfer function of a linear, time-invariant, single-input singleoutput system, $n(\cdot)$ represents a memoryless nonlinearity, and $d(t)$ represents the dither. Different types of dither signals (e.g., sinusoidal, square wave, etc.) have been analyzed in the literature and we

Manuscript received September 7, 1997; revised May 7, 1998. This paper was recommended by Associate Editor L. Fortuna.

The author is with the Bilkent University, Department of Electrical and Electronics Engineering, 06533, Bilkent, Ankara, Turkey.

Publisher Item Identifier S 1057-7122(99)08098-8.

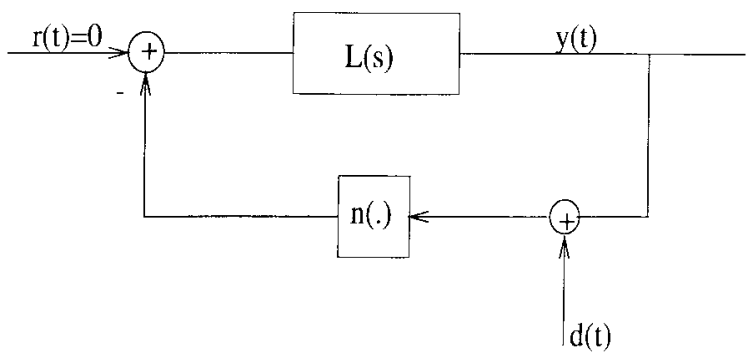

Fig. 1. Application of dither.

consider the following piecewise constant periodic signal in this work:

$$
d(t)=\left\{\begin{array}{cc}
\beta_{1}, & k T<t<\left(\alpha_{1}+k\right) T \\
\beta_{2}, & \left(\alpha_{1}+k\right) T<t<\left(\alpha_{1}+\alpha_{2}+k\right) T \\
\cdots, & \cdots \\
\beta_{l}, & \left(\sum_{i=1}^{l-1} \alpha_{i}+k\right) T<t<(k+1) T \\
k=0,1, \cdots
\end{array}\right.
$$

where $\beta_{i} \in \boldsymbol{R} ; \alpha_{i}>0$ for $i=1,2, \cdots, l ; \Sigma_{i=1}^{l} \alpha_{i}=1$ and $T>0$. It can be shown that with this dither signal, if $n(\cdot)$ satisfies certain conditions and if $T>0$ is sufficiently small, then $n(\cdot)$ could be replaced by the following function $n_{r}(\cdot)$ :

$$
n_{r}(y)=\alpha_{1} n\left(y+\beta_{1}\right)+\alpha_{2} n\left(y+\beta_{2}\right)+\cdots+\alpha_{l} n\left(y+\beta_{l}\right) .
$$

That is, the system in Fig. 1 with the dither signal $d(t)$ given by (1) is equivalent to a similar system without dither in the sense that the trajectories of both systems starting from the same initial condition converge to each other, provided that the nonlinearity $n(\cdot)$ is replaced by $n_{r}(\cdot)$ given by (2) (see e.g., [6], [7], and [12]). If the solutions are bounded, then this property holds provided that $n(\cdot)$ is Lipschitz [6]. The amplitudes (i.e., $\beta_{1}, \beta_{2}, \cdots, \beta_{l}$ ) and the durations (i.e., $\left.\alpha_{1}, \alpha_{2}, \cdots, \alpha_{l-1}\right)$ can be chosen to change the qualitative behavior of the system. The frequency of the dither should be greater than the cut-off frequency of $L(s)$, hence, we assume that $|L(j \omega)| \rightarrow 0$ as $\omega \rightarrow \infty$.

We propose three methods to control the system given in Fig. 1 by using dither.

Changing the System Parameters: Consider the Lur'e system given in Fig. 1 and let the linear block be represented by the transfer function $L(s)=p(s) / q(s)$ where $p(s)$ and $q(s)$ are polynomials. Assume that the nonlinearity $n(\cdot)$ is also a polynomial in $y$ as given below:

$$
n(y)=a_{m} y^{m}+a_{m-1} y^{m-1}+\cdots+a_{1} y+a_{0} .
$$

The dynamical equation of the system in terms of the output variable $y$ can be given as $q(D) y+p(D) n(y)=0$ where $D=(d / d t)$. After the application of the dither signal given by (1), by using (2) and (3) we obtain

$$
n_{r}(y)=\sum_{k=0}^{m} a_{k} \sum_{i=0}^{k}\left(\begin{array}{l}
k \\
i
\end{array}\right) \sum_{j=1}^{l} \alpha_{j} \beta_{j}^{(k-i)} y^{i}
$$

which can be rewritten as

$$
n_{r}(y)=n(y)+\sum_{i=0}^{m-1} r_{i} y^{i}, r_{i}=\sum_{k=1}^{m} a_{k}\left(\begin{array}{l}
k \\
i
\end{array}\right) \sum_{j=1}^{l} \alpha_{j} \beta_{j}^{(k-i)}
$$




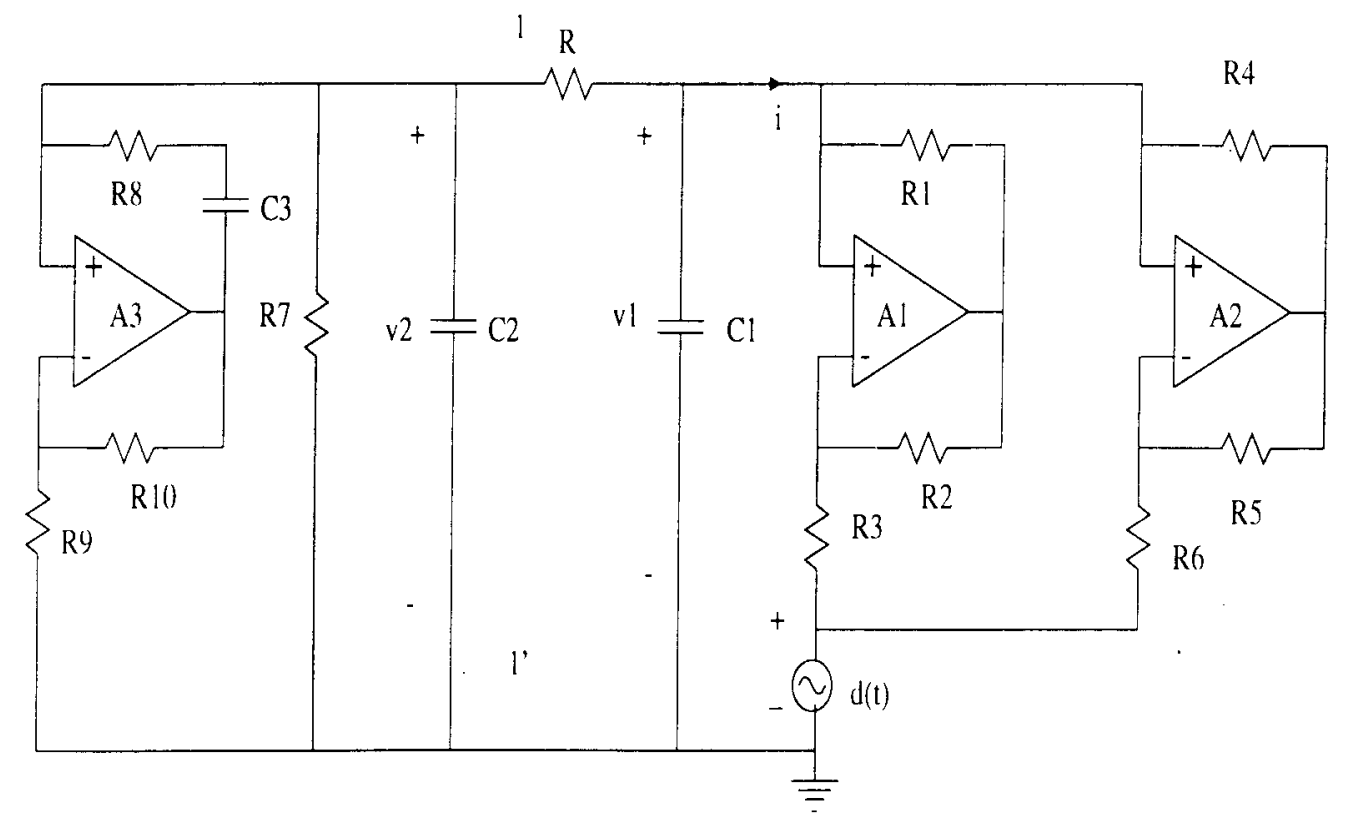

Fig. 2. Wien-bridge chaos generator with dither.

Hence, after the application of the dither, the dynamical equation of the system becomes $q(D) y+p(D) n_{r}(y)=0$. From (3) and (5) it follows that $n_{r}(y)=\Sigma_{j=0}^{j=m} \hat{a}_{j} y^{j}$ where $\hat{a}_{m}=a_{m}$ and $\hat{a}_{j}=a_{j}+r_{j}$, $0 \leq j \leq m-1$. In other words, after the application of dither the modified nonlinearity has the same form as given by (3), but with different coefficients. Hence, if the dynamical behavior of the chaotic system depends on some of these coefficients, we could modify these coefficients by the use of dither and the required dither parameters may be found by using (5). We note that the form of (5) imposes some constraints on $r_{j}$, hence, an arbitrary change in the parameters may not be possible.

The next two methods will be based on the conjecture of Genesio and Tesi. In [3], [4], Genesio and Tesi proposed a conjecture which states that a system in Lur'e form may exhibit chaotic behavior if there exists: 1) a stable limit cycle, 2) an unstable separate equilibrium point, 3) suitable filtering effect on the system, and 4) interaction between the limit cycle and the equilibrium point. The existence and stability properties of the limit cycle can be determined by means of describing function analysis, for details see [3] and [4]. Equilibrium points can be found from the solutions of the following equation:

$$
y+L(0) n(y)=0 .
$$

Stability properties of these equilibrium points can be examined by using linearization. For the predictions made by using the describing function analysis to be reliable, the linear block of the system should have a low-pass characteristic (see, e.g., [5]). Finally, the limit cycle of the system should pass close enough to the unstable equilibrium point. If the (predicted) limit cycle is given as $y(t)=$ $A+B \sin \omega t$, then this condition can be checked analytically by using the interaction parameter $\eta=B /(|A-E|)$, where $E$ is an unstable equilibrium point of the system interacting with the limit cycle. When $\eta$ is close to unity, simulations show that the system may exhibit chaotic behavior and when it is small (near 0.5 in our simulations), the system may exhibit regular motion.

Because of the approximate nature of the analyzing tools and the uncertainty on the value of $\eta$, obviously this conjecture gives neither necessary nor sufficient conditions for the existence of chaos. Now we can state the next two methods.
Changing the Equilibrium Point: In this method, the dither parameters are selected such that the equilibrium points of the chaotic system that interact with the predicted limit cycle are eliminated. This may yield the system to exhibit regular motion. Dither parameters to change equilibrium points can easily be selected by using the following equation whose solutions are the equilibrium points of the dither-applied system:

$$
y+L(0) n_{r}(y)=0
$$

where $n_{r}$ is given by (2). After the parameters are found, the existence and stability of the limit cycle should again be checked.

Changing the Interaction: This method is based on changing the interaction between the equilibrium point and the predicted limit cycle of the original system (i.e., $\eta$ ). Since after the application of dither $\eta$ is a function of $\alpha_{i}, \beta_{i}, i=1, \cdots, l$, by fixing the values of some of these dither parameter beforehand, other dither parameters can be found numerically. After the parameters are found, the existence and stability of the limit cycle should again be checked.

At this point, we compare the effect of dither with the effect of a possible output feedback scheme. Since the effect of dither is to change the nonlinearity $n(\cdot)$ to $n_{r}(\cdot)$ given by (2), obviously if an arbitrary nonlinear output feedback is allowed, then the same effect may be obtained. However, such an output feedback requires output measurement, which may contain measurement errors, a nonlinear operation, which may complicate the realization of the controller and may amplify the errors, and a feedback path, which may also complicate the realization. On the other hand, application of dither, if physically possible, is a much simpler scheme and does not require any measurement. Obviously, a linear feedback may not produce the same effect since it does not contain higher order terms [see (5)]. Also note that, in case of linear feedback, the freedom to change the equilibrium points is limited as compared to the case of dither. To see this, assume that $n(\cdot)$ has the form given by (3) with $a_{0}=0$. It follows from (6) that $y=0$ is always an equlibrium point before and after the linear feedback. However, with the application of dither we have more degrees of freedom to change the equilibrium points (7), (4). The same conclusions may hold if the nonlinearity is not of the form given by (3), e.g., a piecewise linear nonlinearity. 


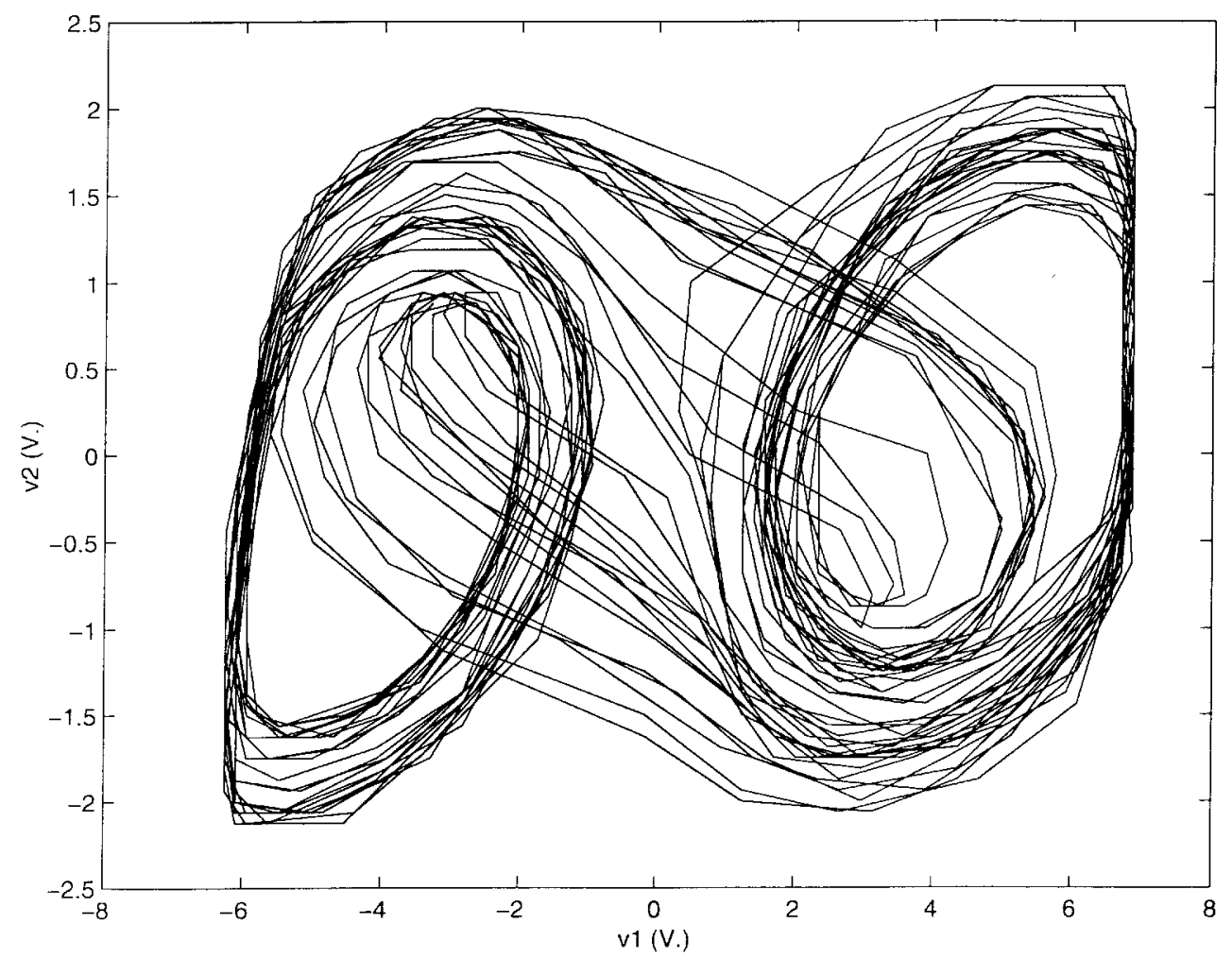

Fig. 3. Double scroll when dither is not applied.

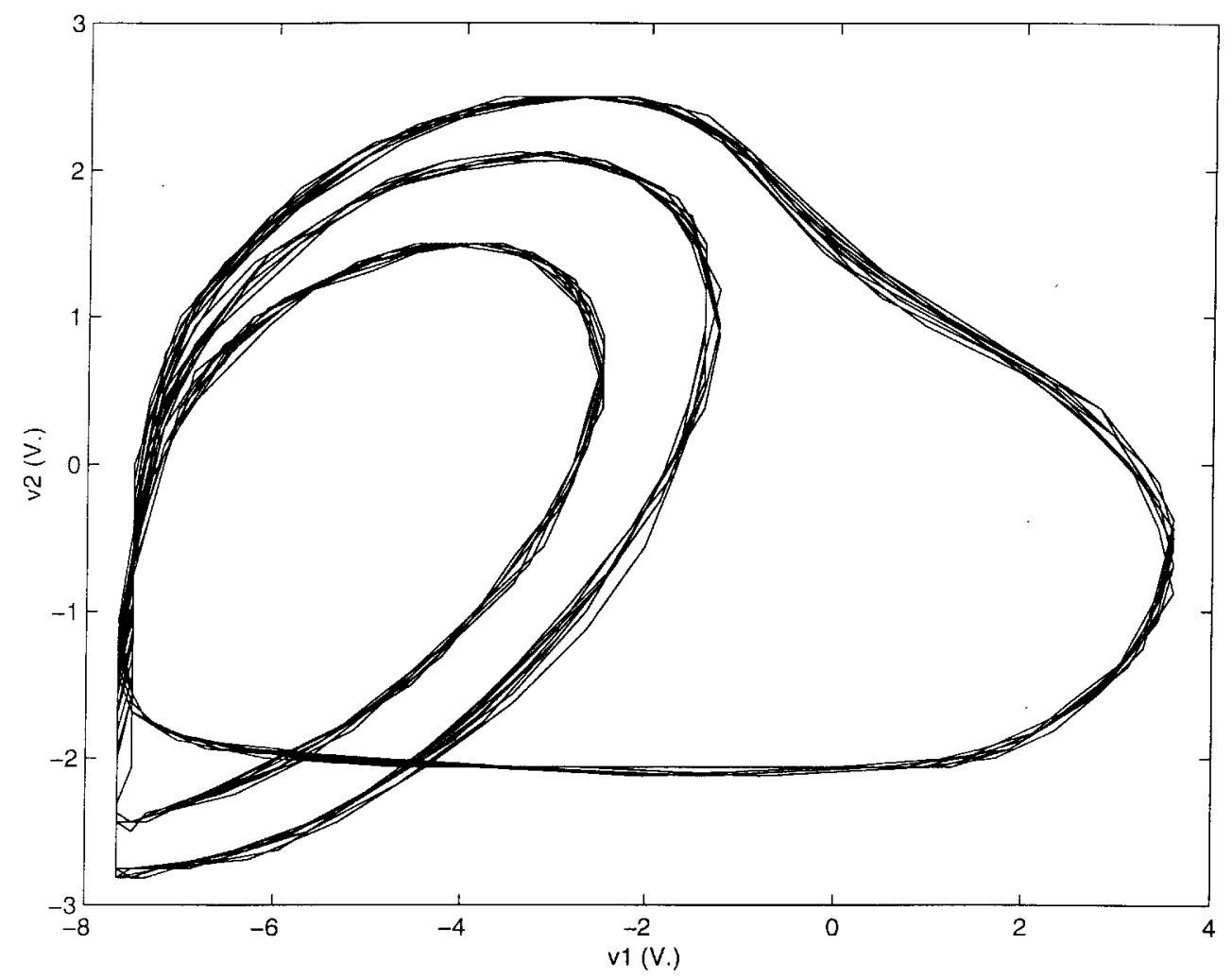

Fig. 4. Period-three limit cycle when $\beta_{1}=320 \mathrm{mV}, \beta_{2}=220 \mathrm{mV}$.

\section{EXPERIMENTAL RESULTS}

In this section we will present some experimental results concerning the application of dither to a chaotic electronic circuit. For such a circuit we consider the (Wien-bridge-based) RC chaotic circuit proposed in [8] and [9], which is shown in Fig. 2. In this figure $d(t)$ represents a dither signal, which is a voltage source in our case. When $d(t)=0$, this circuit may realize the well-known Chua's circuit and this mode of operation is called the "passive mode" [9]. It is also 


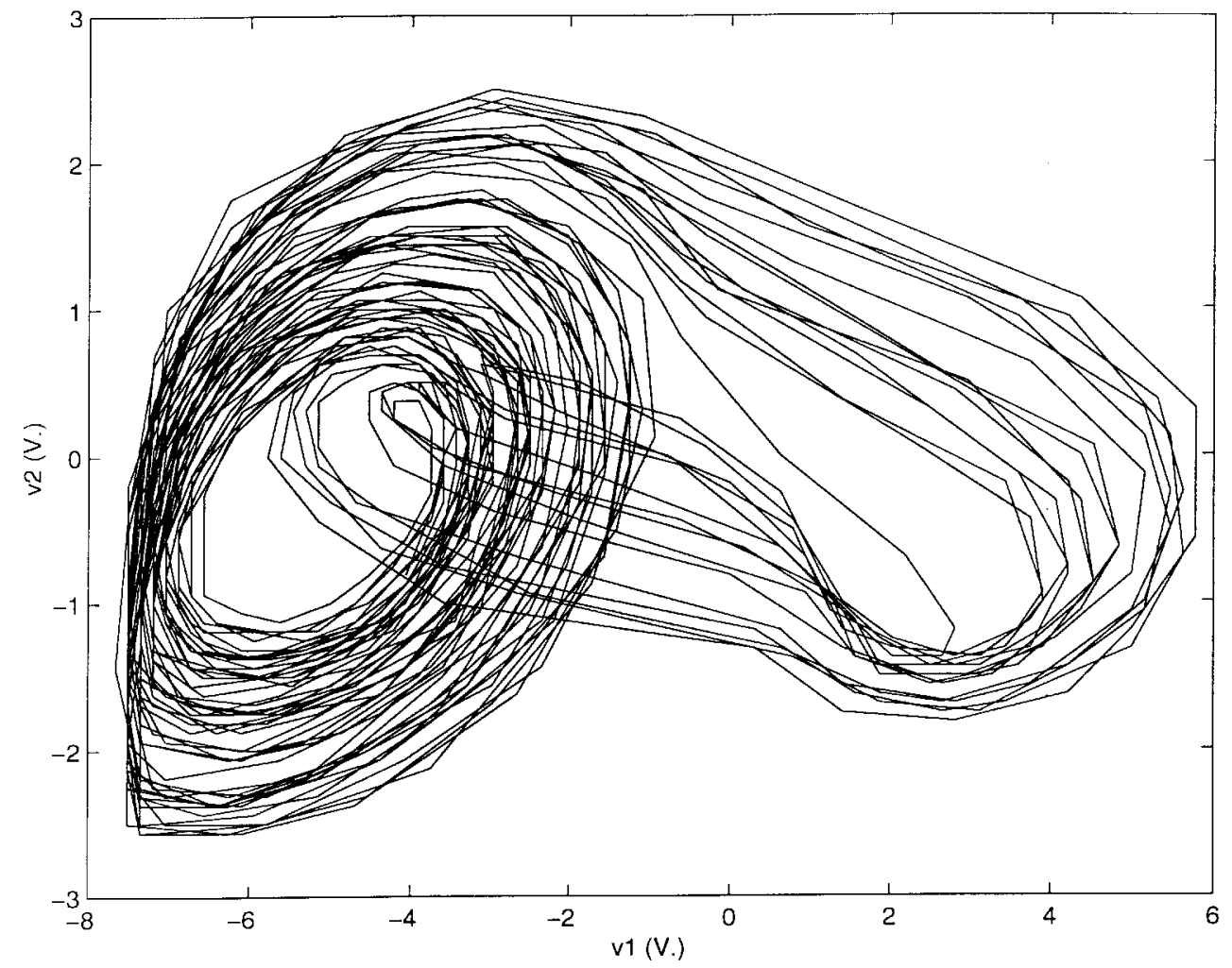

Fig. 5. Single scroll when $\beta_{1}=150 \mathrm{mV}, \beta_{2}=50 \mathrm{mV}$.

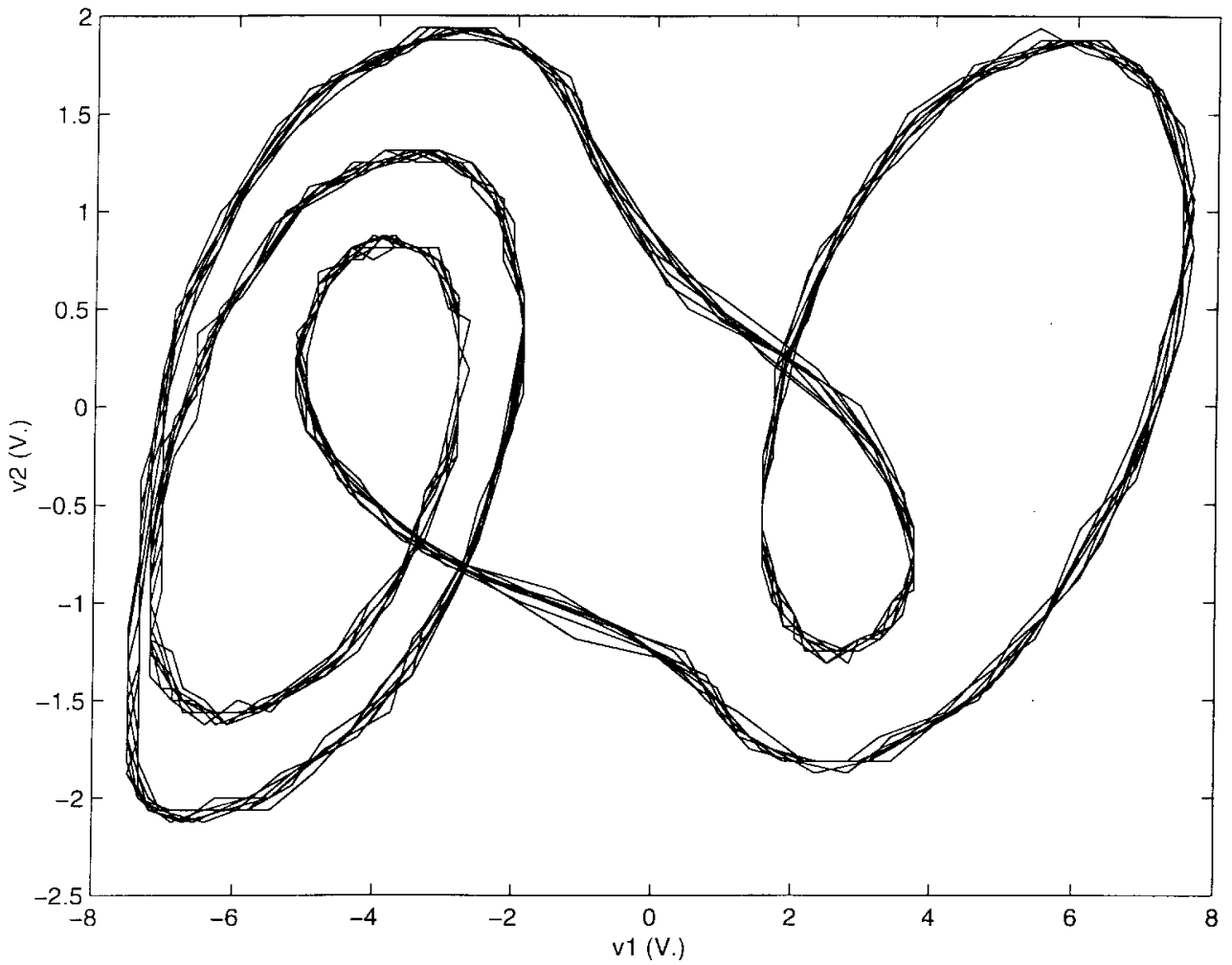

Fig. 6. Limit cycle when $\beta_{1}=100 \mathrm{mV}, \beta_{2}=0 \mathrm{mV}$.

possible first to tune the Wien bridge to oscillatory mode and then observe chaotic behavior by using the coupling resistor $R$, [9]. In both modes of operations, various forms of chaotic behaviors (e.g., single scroll, double scroll) can be observed.
In Fig. 2, the operational amplifiers $A_{1}$ and $A_{2}$ and the related resistors $R_{1}, \cdots, R_{6}$ realize the three-segment resistor called the Chua diode, which is also used in the standard implementation of the Chua's circuit [11]. As in [11], we chose $R_{1}=R_{2}=220 \Omega$, 
$R_{3}=2.2 \mathrm{k} \Omega, R_{4}=R_{5}=22 \mathrm{k} \Omega, R_{6}=3.3 \mathrm{k} \Omega$. Note that this part represents a voltage controlled resistor of the form $i=g\left(v_{1}\right)$ when $d(t)=0[11]$ and with the dither, this relation becomes $i=g\left(v_{1}-d\right)$ (cf. Fig. 1). The circuit to the left of $1-1^{\prime}$ is a Wien bridge circuit and we chose the related parameters as $R_{7}=R_{8}=R_{9}=100 \Omega$, $R_{10}=208 \Omega, C_{2}=C_{3}=220 \mathrm{nF}$. (We note that $R_{10}=200 \Omega$ is the theoretical value to start oscillations for the Wien bridge when $R$ is open-circuited). The resistors $R_{10}$ and $R$ are potentiometers and while $R_{10}$ is used to tune the Wien bridge part, $R$ is used to obtain chaotic behavior. For $R=1980 \Omega$ and $C_{1}=1.5 \mathrm{nF}$ and when $d(t)=0$, this circuit exhibits a double-scroll behavior, as shown in Fig. 3. We note that all operational amplifiers are LF 351N (biased by $\pm 9 \mathrm{~V}$ ) in our experiments, however, equivalent ones could also be used.

For the dither signal we used the waveform given by (1) with $l=2$ and $\alpha_{1}=\alpha_{2}=0.5$. This type of waveform could easily be generated by using the standard square wave generators with offsets. By arranging the magnitudes $\beta_{1}, \beta_{2}$ we observed various behaviors and, here, we report only four such results, due to space limitations. In these experiments we chose the frequency of the dither signal as $f=100 \mathrm{kHz}$, and observed the same behavior for $f \geq 100 \mathrm{kHz}$ (and in some cases for $f \geq 50 \mathrm{kHz}$ ).

1) For $\beta_{1}=320 \mathrm{mV}, \beta_{2}=220 \mathrm{mV}$, we observed a period-three limit cycle, as shown in Fig. 4.

2) For $\beta_{1}=150 \mathrm{mV}, \beta_{2}=50 \mathrm{mV}$, we observed a single-scroll chaos as shown in Fig. 5 .

3) For $\beta_{1}=100 \mathrm{mV}, \beta_{2}=0 \mathrm{mV}$, we observed a limit cycle as shown in Fig. 6.

4) For $\beta_{1}=60 \mathrm{mV}, \beta_{2}=-40 \mathrm{mV}$, we observed a double-scroll similar to the one shown in Fig. 3.

Various comments are in order. We generated the dither signals by first obtaining a square wave with zero offset $(100 \mathrm{mV}$ peak to peak in our experiments) and then by changing the offset. We observed similar behaviors by increasing peak-to-peak voltage, provided that the frequency is also increased. For example, when $\beta_{1}$ is increased and $\beta_{2}$ is decreased by $150 \mathrm{mV}$ in the experiments given above, the same behaviors are observed, provided that $f \geq 450 \mathrm{kHz}$.

In all experiments, we first observed the $v_{1}-v_{2}$ characteristics in an analogue oscilloscope in $X-Y$ mode. Then the same figure is obtained in a digitizing oscilloscope (HP 54600B). After storing the screen in the memory of the oscilloscope, the data is transferred to a computer by using an HB-IB bus. Since the important information is $v_{1}-v_{2}$ graphics, we do not present individual signals $v_{1}(t)$ and $v_{2}(t)$.

We also note that various simulation results concerning the application of dither to some well-known chaotic systems, such as Chua's circuit and the Duffing oscillator, can be found in [10] and [12].

\section{CONCLUSION}

In this paper we proposed the use of an additive dither signal for the control of chaotic systems in the Lur'e form. Many types of dither signals could be used and we considered piecewise constant dither signal for simplicity. Dither has the effect of modifying the nonlinearity and by using this property it may be possible to control the dynamic behavior of chaotic systems. By exploiting this property, we proposed three methods to control such chaotic systems, (i.e., to switch between the chaotic and regular trajectories). We also presented some experimental results.

The dither is an external signal applied before the nonlinear block. Hence, its application does not require any continuous measurement, which is the case in the feedback control schemes. Therefore, the main advantage of the application of dither is its simplicity. However, the technique is not general, its effect is limited (2), and it could be applied to systems in Lur'e form. In chaotic electronic circuits, the output variable is usually either a voltage or a current variable and, for such cases, the dither source is either an independent voltage or current source, which could easily be realized and applied.

In this work, we considered the piecewise constant dither signals. However, different periodic signals (e.g., sinusoidal signals) may also be used as dither signal. The effect of dither on systems not in the Lur'e form may also be analyzed. These points require further research.

\section{REFERENCES}

[1] E. Ott, C. Grebogi, and J. A. Yorke, "Controlling chaos," Phys. Rev. Lett., vol. 64, no. 11, pp. 1196-1199, 1990.

[2] G. Chen, Control and synchronization of chaotic systems. Available FTP: ftp.egr.uh.edu/pub/TeX/chaos.tex.

[3] R. Genesio and A. Tesi, "Chaos prediction in nonlinear feedback systems," Proc. Inst. Elect. Eng., vol. 138, pt. D, pp. 313-320, July 1991.

[4] _ "Harmonic balance methods for the analysis of chaotic dynamics in nonlinear systems," Automatica, vol. 28, no. 3, pp. 531-548, 1992.

[5] D. P. Atherton, Nonlinear Control Engineering. New York: Van Nostrand Reinhold, 1982.

[6] A. M. Steinberg and I. Kadushin, "Stabilization of nonlinear systems with a dither control," J. Math. Anal. Appl., no. 43, pp. 273-284, 1973.

[7] G. Zames and N. A. Shneydor, "Dither in nonlinear systems," IEEE Trans. Automat. Contr., vol. 21, pp. 660-667, Oct. 1976.

[8] Ö. Morgül, "Inductorless realization of Chua oscillator," Electron. Lett., vol. 31, no. 17, pp. 1403-1404, 1995.

[9] _ "Wien bridge based RC chaos generator," Electron. Lett., vol. 31, no. 24, pp. 2058-2059, 1995.

[10] Ö. Morgül and U. Ersoy, "On the application of dither for controlling some chaotic systems," in Proc. ECCTD'97, Budapest, Hungary, vol. 3, pp. 1216-1221, Aug. 1997.

[11] M. P. Kennedy, "Three steps to chaos-Part 2: A Chua's circuit primer," IEEE Trans. Circuits Syst., vol. 40, pp. 657-675, Oct. 1993.

[12] U. Ersoy, Application of dither and observer based state feedback in the control of chaotic systems, M.S. thesis, Bilkent Univ., Ankara, Turkey, Aug. 1996.

\section{Corrections to "Separation Conditions and Approximation of Continuous-Time Approximately Finite Memory Systems"}

\author{
Irwin W. Sandberg
}

The following errors, introduced by the staff associate editor, occurred in the above paper. ${ }^{1}$

On page 822, the sentence following Statements 1) and 2) in Lemma 1 should read:

"Continuing with the proof of Theorem 1, suppose that 1) of the theorem holds and choose any positive $\alpha$."

Manuscript received September 3, 1999

The author is with the Department of Electrical and Computer Engineering, The University of Texas at Austin, Austin, TX 78712 USA.

Publisher Item Identifier S 1057-7122(99)09621-X.

${ }^{1}$ I. W. Sandberg, IEEE Trans. Circuits Syst. I, vol. 46, pp. 820-826, July 1999. 